\title{
NIVEL DE APROBACIÓN DE LAS COMPETENCIAS PROFESIONALES INICIALES DE LOS ESTUDIANTES, APLICADAS POR DOCENTES DE PRÁCTICA CLÍNICA. EXPERIENCIA UNIVERSIDAD DE PLAYA ANCHA
}

\author{
LEVEL OF APPROVAL FOR THE INITIAL PROFESSIONAL COMPETENCES OF \\ STUDENTS ACCORDING TO THE TEACHERS GUIDE FOR PROFESSIONAL PRACTICE: \\ EXPETIANCIA AT THE UNIVERSITY OF PLAYA ANCHA
}

\section{Rosa Eleonora Chaparro ${ }^{1}$}

\section{Resumen}

El estudio está orientado a mejorar la calidad de la formación profesional en el área de la salud, de los Terapeutas Ocupacionales de la Universidad de Playa Ancha de Ciencias de la Educación, Valparaíso, para tal efecto se determinan las competencias profesionales implícitas en el diseño del Plan de Estudios de formación profesional de Terapia Ocupacional, y se describe el nivel de aprobación de los Profesores Guía de Práctica Profesional de los egresados del Plan de Estudios el año 2004, respecto de su formación en las distintas áreas de la salud donde ellos realizan su práctica profesional.

Los resultados describen el logro del desempeño de las competencias profesionales de Terapia Ocupacional, mostrando áreas específicas de la disciplina factibles de mejorar.

\section{Palabras clave:}

Competencias profesionales, Terapia Ocupacional.

\begin{abstract}
The study aims to improve the quality of vocational training in the area of health, occupational therapists at the University of Playa Ancha Science Education, Valparaíso; for this purpose are determined skills implicit in the design of curriculum training of Occupational Therapy, and describes the level of approval of the Faculty Practice Guide of the graduates curriculum 2004, for training in different areas of health where they perform their professional practice.

The results describe the achievement of the performance of the professional skills of Occupational Therapy; showing specific areas of the discipline may be improved.
\end{abstract}

\section{Key words:}

Professional Competencies, Occupational Therapy.

\footnotetext{
Terapeuta Ocupacional. Académico Universidad de Playa Ancha. Avenida Playa Ancha № 850, VALPARAíSO. TELEFONO: (56) (32) 2500228
} 


\section{INTRODUCCIÓN}

En el marco de los esfuerzos por mejorar la calidad de la educación superior en el área de la salud, se diseñó por primera y única vez en el Departamento Disciplinario de Ciencia de la Ocupación Humana de la Universidad de Playa Ancha de Valparaíso (UPLA), un estudio en el que se plantea verificar las competencias profesionales del ejercicio del rol del Terapeuta Ocupacional implícitas en el Plan de estudios de la carrera de Terapia Ocupacional del año 2000 de la UPLA, en las distintas áreas de la salud donde este profesional se desempeña.

Para que los Terapeuta Ocupacionales puedan cumplir con su misión y responder a las expectativas de las necesidades de prestaciones de salud de la población, es imprescindible que se cumpla con la adquisición de las competencias profesionales para el desempeño laboral declaradas en su programa de estudios, e implementadas en el proceso de formación profesional (Irigoin, M. y Vargas, F. 2002).

La habilitación para el desempeño profesional se considera alcanzada cuando el estudiante de la carrera de Terapia Ocupacional dispone de los conocimientos y de las capacidades suficientes para enfrentar las tareas y resolver los problemas propios del trabajo profesional, lo que se da por hecho una vez que ha concluido el proceso de formación académica, que es al término de su formación teórico-práctica; es decir cuando ha aprobado todo el plan de estudios.

Guy Le Boterf (2013), reconocido experto en desarrollo y gestión de competencias, define la diferencia entre los conceptos competente y tener competencia; al respecto refiere que muchos estudiantes aprenden contenidos y aprueban planes de estudios, considerándose capacitados para la vida laboral, sin embargo carecen de las habilidades necesarias para un óptimo desempeño en el área de su profesión, agrega que las Universidades deben hacerse cargo de esta situación "pues deben cambiar su visión sobre cultura e identificar cuáles son los procesos que permitirán desarrollar a los estudiantes, el conjunto de acciones e interacciones para hacer frente a una situación real" (Le Bortef,G. 2013).

Para que la calidad de la formación profesional quede de manifiesto, se requiere de un aporte científico que ofrezca evidencias de que la preparación teórica de los egresados de un programa de estudios de pre-grado, como ocurre en este caso; cuentan con los conocimientos y desarrollo de habilidades mínimas para cumplir funciones que le son propias con los pacientes / usuarios (Latrach, Soto, González, Caballero \& Inalaf. 2009).
El presente estudio diseña un instrumento de evaluación de las competencias profesionales iniciales de los estudiantes de la Carrera de Terapia Ocupacional de la Universidad de Playa Ancha, sede Valparaíso, que han concluido su plan de estudios, y lo aplica a los Profesores Guía de Práctica Profesional, quienes al responderlo, juzgarán la calidad y de la preparación académica de los estudiantes en las áreas de formación profesional específicas de la especialidad de la salud en que ellos trabajan.

Este instrumento ha sido confeccionado a partir del perfil profesional del Terapeuta Ocupacional, declarado en el Programa de formación profesional de esta Casa de Estudios. Ha sido sometido a los procedimientos de validación y confiabilidad y se ha administrado en forma de entrevista al universo de Profesores Guía de Práctica Profesional que supervisaron a lo menos, tres estudiantes de la Carrera durante el año 2004.

A continuación, se han aplicado las pruebas estadísticas para determinar la frecuencia del grado de aprobación de las competencias profesionales iniciales de los alumnos que concluyeron su formación el año 2004 ,y se describen. Luego se ha determinado el grado de aprobación de las competencias profesionales iniciales de los egresados, por área de especialidad de la Terapia Ocupacional, y se describen. Finalmente, se determina el nivel de aprobación por competencia profesional por cada egresado(a), el cual también se describe.

En un segundo análisis se ha descrito el nivel de aprobación de los Profesores Guía según los años de ejercicio como docente de la educación superior, y los años de ejercicio de la profesión.

\section{Marco Metodológico}

\section{Objetivo General}

Contribuir al mejoramiento de la calidad de la gestión del aprendizaje, a través de la definición y evaluación de las competencias profesionales implícitas en el Plan de Estudios de la carrera de Terapia Ocupacional de la Universidad de Playa Ancha, sede Valparaíso, 2004.

\section{Objetivos Específicos}

Identificar las competencias iniciales, propias del ejercicio profesional, determinadas por el Plan de Estu- 
dios y definidas en el perfil del Terapeuta Ocupacional, como garantía de identidad y eficiencia para ejercer la profesión.

Describir las competencias profesionales iniciales del desempeño profesional de los egresados del programa de formación profesional de Terapia Ocupacional de la UPLA, 2004.

Describir cuantitativamente, el nivel de logro de las competencias profesionales iniciales de los egresados el año 2004 del programa de formación profesional de Terapia Ocupacional, en las áreas de la salud, definidas en el Plan de Estudios, desde el juicio de experiencia laboral de los Profesores Guía de Práctica Profesional.

Describir cuantitativamente, el nivel de logro de cada una de las dimensiones de la variable competencias profesionales iniciales de los egresados el año 2004 del programa de formación profesional de Terapia Ocupacional, desde el juicio de experiencia laboral de los Profesores Guía de Práctica Profesional.

Describir cuantitativamente los resultados por área de especialidad en salud de la Terapia Ocupacional, respecto del nivel de logro de las competencias profesionales iniciales propias del ejercicio profesional de los egresados el año 2004 del programa de formación profesional de Terapia Ocupacional, desde el juicio de experiencia laboral de los Profesores Guía de Práctica Profesional.

Describir cuantitativamente los resultados para cada dimensión de la variable, competencias profesionales, por área de especialidad en salud de la Terapia Ocupacional, respecto del nivel de logro de las competencias profesionales iniciales propias del ejercicio profesional de los egresados el año 2004, del programa de formación profesional de Terapia Ocupacional, desde el juicio de experiencia laboral de los Profesores Guía de Práctica Profesional.

Describir cuantitativamente, el nivel de logro de la formación de las competencias profesionales iniciales propias del ejercicio profesional de los egresados el año 2004, del programa de formación profesional de Terapia Ocupacional, desde el juicio de experiencia laboral de los Profesores Guía de Práctica Profesional, según los años de servicio de este profesional en el desempeño de la profesión.

Describir cuantitativamente, el nivel de logro de la formación de las competencias profesionales iniciales propias del ejercicio profesional de los egresados el año 2004, del programa de formación profesional de Terapia Ocupacional, desde el juicio de experiencia laboral de los Profesores Guía de Práctica Profesional, según los años de ejercicio como docente de la Educación Superior de este profesional.

El Universo corresponde a 23 Profesores Guía de Práctica Profesional del Programa de Formación Profesional de Terapia Ocupacional de la UPLA, que han prestado sus servicios profesionales en los distintos Centros de Práctica Profesional en las áreas de la Salud Física Adulto, Salud Mental y Psiquiatría, Pediatría y Geriatría y Gerontología, en convenio con la UPLA, en el año 2004. Además, estos Profesores Guía han tenido a su cargo al menos tres alumnos en práctica durante ese año.

\section{Variables De Estudio}

Nivel de aprobación de los Profesores Guías de Práctica Profesional. Para efectos de esta investigación, queda definida la aprobación de los Profesores Guía, como el resultado del grado o nivel de la aceptación que estos determinan, toda vez que el desempeño del trabajo del estudiante en la Práctica Profesional le permita resolver los problemas de salud abordados, desarrollando en forma efectiva el repertorio de evaluaciones e intervenciones de tratamiento de Terapia Ocupacional en relación con el desarrollo humano. Esta aceptación deriva de las competencias profesionales del Profesor Guía y de la efectividad del aprendizaje de los alumnos en el proceso de su formación profesional. Esta variable se operacionaliza a través de la Encuesta de Opinión de los Profesores Guía de Práctica Profesional, acerca del desempeño profesional inicial de los estudiantes de la Carrera de Terapia Ocupacional de la UPLA, 2004.

Competencias Profesionales Iniciales de los estudiantes de la Carrera de Terapia Ocupacional de la Universidad de Playa Ancha sede Valparaíso, en el área de la Formación Profesional durante el año 2004. Para efectos de esta investigación queda definida como "el conjunto de saberes (saber, saber hacer, saber estar y saber ser; conocimientos, procedimientos y actitudes) que combinados, coordinados e integrados en la Práctica Profesional" (Cejas y Pérez, 2003), de los egresados de la carrera de Terapia Ocupacional con menos de un año de práctica los capacita para actuar con eficacia en una situación profesional. Esta variable se operacionaliza a través del logro de las Competencias Profesionales Iniciales, de los egresados de la Carrera de Terapia Ocupacional de la Universidad de Playa Ancha de Ciencias de la Educación que hayan cursado la asignatura de Práctica Profesional el año 2004. Las Competencias 
Profesionales son: Evaluación (de la situación de discapacidad del usuario), Planificación (de la intervención), Intervención (al usuario), Solución de problemas, y el Profesionalismo / administrativo.

\section{Instrumento}

Se utilizó una entrevista estructurada con pauta dirigida de tipo mixto, con un total de 61 preguntas, de las cuales 56 son cerradas (de alternativa) y 05 abiertas (de opinión), aplicada a los Profesores Guías de Práctica Profesional.

Tiene una escala de clasificación jerárquica con la que el Profesor Guía asignó el grado de aceptación de la competencia profesional. El sistema de medición es del tipo Escala de Lickert, donde los valores determinados para las variables van del 1 al 5; siendo el nivel 3 el que da la competencia por lograda, cuando aprecia que "Puede desarrollar esta tarea sin asistencia y/o supervisión", y el 5 cuando aprecia que "Puede desarrollar esta tarea con una velocidad y calidad más que aceptables, y con iniciativa y adaptabilidad para situaciones problemáticas".

La entrevista estructurada consta de cinco dimensiones, la Evaluación, la Planificación, la Intervención o Tratamiento, la Solución de Problemas y el Profesionalismo / Administrativo.

La validez del instrumento es de un $0,96 \%$ y la Confiabilidad es de 0,9867 puntos.

\section{Procedimiento}

A partir del Perfil Profesional y del Plan de Estudios del Programa de Formación Profesional, se establecen las dimensiones de la variable Competencias Profesionales iniciales, y se diseña un instrumento de evaluación de las mismas. Se realizaron procedimientos de validez y confiabilidad de este.

Se tomó contacto telefónico con los Profesores Guía de Práctica Profesional (académicos contratados a honorarios), se les informó de la investigación y se confirmó su participación en un $100 \%$ de ellos. A continuación se les visitó en sus puestos de trabajo, se aplicó un certificado de consentimiento informado que asegura la confidencialidad de sus respuestas, y se administró el instrumento de evaluación en forma de entrevista. Ellos a través de sus respuestas juzgaron la calidad de la formación académica de los estudiantes en las áreas de formación profesional específicas de su especialidad.

Posteriormente, se aplicaron pruebas estadísticas descriptivas para determinar el nivel de aprobación de las competencias profesionales iniciales de los egresados el año 2004, y se describieron.

En un segundo análisis, se ha descrito el nivel de logro establecido por la población entrevistada, según el género, los años de ejercicio como docente de educación superior, y según los años de ejercicio de la profesión.

Para el análisis de la información proveniente de las respuestas abiertas del instrumento, se codificaron los datos en dos niveles; siendo la Unidad de análisis determinada, los párrafos. Las categorías determinadas fueron las variables de estudio relativas a las competencias profesionales y por último, estas categorías se codificaron del siguiente modo, a saber: 1. -Evaluación, 2. -Planificación, 3. -Intervención, 4. -Solución de problemas, 5.- Profesionalismo administrativo y 6. -Otras.

En el segundo nivel se realizó la interpretación del significado de los datos de las categorías obtenidas en el primer nivel por Grinnell (1997), y Berg (1995), en Hernández Sampieri, R.; Fernández Collado, C. y Baptista Lucio, P. (2003), equiparándolas e integrándolas en dos grandes temas de interés para la investigación que son las Fortalezas y las Debilidades de la Práctica. Se determinó la frecuencia de mención, sin hacer relación entre las categorías.

\section{Resultados}

\section{De los fundamentos teóricos}

A partir del análisis de la bibliografía y a la luz de la discusión de los resultados, este estudio logra establecer lo siguiente:

Respecto del primer objetivo específico que era identificar las competencias iniciales, propias del ejercicio profesional, determinadas por Plan de Estudios y definidas en el perfil profesional, que desempeña el estudiante de Terapia Ocupacional de la UPLA como garantía de la identidad y eficiencia para ejercer la profesión; la presente investigación las define como Evaluación; Planificación, Intervención, Solución de problemas y Profesionalismo administrativo. 
Se hace necesario destacar que tal definición además de concordar con la revisión de la literatura, es a la vez el quehacer del Terapeuta Ocupacional en las distintas áreas de la salud donde los estudiantes en práctica desarrollan su trabajo de campo al ejecutar intervenciones propias de la disciplina que conducen a resolver los problemas que presentan los usuarios del sistema.

Respecto del segundo objetivo específico, que era determinar y describir las competencias profesionales iniciales del desempeño laboral de los estudiantes de Terapia Ocupacional de la UPLA, definidas en el Plan de Estudios y en el perfil profesional; este estudio a partir de la revisión bibliográfica, las define del siguiente modo:

- evaluación; se refiere al proceso de obtención, interpretación y documentación de los datos necesarios para la intervención profesional, sumado a los recursos aportados por el Terapeuta Ocupacional y el usuario, de las actividades que se pueden utilizar. Este proceso de evaluación incluye información acerca de la patología o discapacidad existente y sus efectos sobre la vida del paciente, también debe incluir datos sobre las destrezas del usuario o cliente, y antecedentes relacionados con las potencialidades, educación y vocación del mismo, como también, un análisis claro del contexto. Existen dos tipos de evaluación, la especifica, que es una evaluación periódica y continua, y la evaluación inicial.

- planificación, es una descripción realista y detallada de las actividades y métodos a utilizar para lograr las metas acordadas. Está constituida por dos pasos: la identificación de los problemas donde se especifica y esclarece la naturaleza de estos, analizando sus elementos y componentes, y el desarrollo de una solución, que implica clasificar la información recolectada y establecer los objetivos y métodos a través de los cuales se abordarán los problemas. La planificación de la intervención representa una síntesis del conocimiento del Terapeuta Ocupacional con relación al potencial de las actividades, y su relación con los problemas identificados en el usuario para que las acciones determinadas actúen como facilitadoras del crecimiento y desarrollo funcional del cliente.

- intervención, es la vinculación de la acción con el desempeño de las actividades, y con las metas de tratamiento. La intervención actúa sobre el contrato implícito desarrollado entre el Terapeuta Ocupacional y el usuario.
- solución de problemas, la solución de problemas implica las habilidades, las destrezas y el conocimiento teórico del Terapeuta Ocupacional en el desarrollo de cinco pasos básicos en el trabajo con el paciente: la identificación del problema, el desarrollo de una solución, el desarrollo de un plan de acción, la ejecución de un plan de acción, y la evaluación de los resultados.

- profesionalismo administrativo, son las habilidades y destrezas propias y adquiridas por el Terapeuta Ocupacional para participar con vocación de servicio, y una actitud empática en la relación que establece con el usuario.

\section{De los fundamentos empíricos}

A partir del análisis de los resultados, el presente estudio concluye lo siguiente.

Respecto del tercer objetivo específico el nivel de aprobación de los Profesores Guía de Práctica Profesional de las competencias profesionales iniciales de los estudiantes que egresan el año 2004 de la carrera de Terapia Ocupacional que dicta la UPLA, la presente investigación, considerando los casos válidos, que se adscriben al criterio aprobado, a partir de la calificación 3 en cada ítem, ha determinado que el 80,56\% de la población las califica como logradas. Este criterio queda ratificado también en el análisis de la media obtenida para la escala total, cuya ponderación alcanza el puntaje 3,439 de la escala de medición, con una desviación estándar de 6972 puntos.

Respecto del cuarto objetivo específico cuya meta era verificar y describir cuantitativamente el nivel de aprobación de los Profesores Guía de Práctica Profesional respecto de cada una de las dimensiones de las competencias profesionales iniciales de los estudiantes que egresan el año 2004 de la carrera de Terapia Ocupacional de la UPLA, la presente investigación, considerando los casos válidos, que se adscriben al criterio aprobado, a partir de la calificación 3 en cada ítem, los Profesores Guía de Práctica Profesional, han determinado que estas han sido logradas, en cada una de sus dimensiones, tal como lo muestra la siguiente tabla: 
Tabla $N^{\circ} 1$

Descripción de las medias y la desviación estándar para cada una de las dimensiones de la variable "competencia profesional"

\begin{tabular}{|c|c|c|c|c|c|}
\hline & $\begin{array}{c}\mathrm{N}^{\circ} \\
\text { Profesores }\end{array}$ & Mínimo & Máximo & Media & $\begin{array}{c}\text { Desviación } \\
\text { Estándar }\end{array}$ \\
\hline Evaluación & 23 & 2,1 & 4,8 & 3,430 &, 7516 \\
\hline Planificación & 23 & 1,8 & 4,6 & 3,165 &, 8942 \\
\hline Intervención & 23 & 1,2 & 5,0 & 3,348 &, 8339 \\
\hline Solución /Problemas & 23 & 1,9 & 4,8 & 3,217 &, 8066 \\
\hline $\begin{array}{c}\text { Profesionalismo/ } \\
\text { Administrativo }\end{array}$ & 23 & 2,8 & 5,0 & 4,035 &, 7209 \\
\hline Escala Total & 23 & 2,3 & 4,8 & 3,439 &, 6972 \\
\hline Valid N (listwise) & 23 & & & & \\
\hline
\end{tabular}

Considerando que los sujetos tienen el mismo nivel de competencia en las distintas dimensiones de la variable, a excepción de "Profesionalismo / Administrativo", se realiza un análisis estadístico de comparación de las medias de las escalas donde se formula la hipótesis estadística nula de igualdad de los puntajes medios de las escalas, aplicando el Test de comparación de medias para muestras relacionadas a un nivel de significancia al 5\%, y se construye la Tabla con los resultados.

Tabla $\mathrm{N}^{\circ} 2$

Igualdad de la medias para el test de comparación de muestras relacionadas a un 5\% de significancia

\begin{tabular}{|c|c|c|c|c|c|c|}
\hline & Media & 3,17 & 3,22 & 3,35 & 3,43 & \\
\hline & & $\mathrm{P}$ & $\mathrm{S}$ & $\mathrm{I}$ & $\mathrm{E}$ & $\mathrm{P} \mathrm{A}$ \\
& & $\mathrm{L}$ & $\mathrm{P}$ & $\mathrm{N}$ & $\mathrm{V}$ & $\mathrm{R} \mathrm{D}$ \\
$\mathrm{M}$ & & $\mathrm{A}$ & $\mathrm{R}$ & $\mathrm{T}$ & $\mathrm{a}$ & $\mathrm{O} \mathrm{M}$ \\
$\mathrm{e}$ & $\mathrm{N}$ & $\mathrm{O}$ & $\mathrm{E}$ & $\mathrm{I}$ & $\mathrm{F} . \mathrm{I}$ \\
$\mathrm{d}$ & $\mathrm{I}$ & $\mathrm{B}$ & $\mathrm{R}$ & $\mathrm{u}$ & $\mathrm{N}$ \\
$\mathrm{i}$ & & $\mathrm{F}$ & $\mathrm{L}$ & $\mathrm{V}$ & $\mathrm{a}$ & $\mathrm{I}$ \\
$\mathrm{a}$ & & $\mathrm{I}$ & $\mathrm{E}$ & $\mathrm{E}$ & $\mathrm{C}$ & $\mathrm{S}$ \\
& & $\mathrm{C}$ & $\mathrm{M}$ & $\mathrm{N}$ & $\mathrm{i}$ & $\mathrm{T}$ \\
& & $\mathrm{A}$ & $\mathrm{A}$ & $\mathrm{C}$ & $\mathrm{O}$ & $\mathrm{R}$ \\
& & $\mathrm{C}$. & $\mathrm{S}$ & & $\mathrm{n}$ & $\mathrm{A}$ \\
\hline 3,17 & Planificación & & $=$ & $=$ & $<$ & $<$ \\
\hline 3,22 & Solución De Problemas & & & $=$ & $=$ & $<$ \\
\hline 3,35 & Intervención & & & & $=$ & $<$ \\
\hline 3,43 & Evaluación & & & & & $<$ \\
\hline
\end{tabular}


La comparación de las escalas en sus Medias, define la ambigüedad al ser el resultado de Profesionalismo / Administrativo menor al 5\% del de competencia, en comparación con las otras dimensiones de las variables. En otras palabras, bajo la hipótesis estadística nula de igualdad de los puntajes medios de las escalas, esta se rechaza para la escala "Profesionalismo / Administrativo, en comparación con el resto de las escalas.
En cuanto a la distribución de la frecuencia de mención de las respuestas abiertas, codificadas de acuerdo al análisis del contenido referido, por los Profesores Guía para cada una de las variables de la Competencia profesional, los resultados, en cuanto a las Fortalezas desarroIladas por los estudiantes, destacan las siguientes:

Tabla No 3

Fortalezas de las competencias profesionales

\begin{tabular}{|c|c|c|}
\hline Competencia & $\begin{array}{l}\mathrm{N}^{\mathrm{o}} \\
\text { Profesores }\end{array}$ & Respuesta Abierta \\
\hline \multirow{3}{*}{ Evaluación } & 04 & $\begin{array}{l}\text { Inicialmente les cuesta integrar conocimientos para fundamentar la elección de } \\
\text { pautas de evaluación y su interpretación, pero logran alcanzar el objetivo. Desta- } \\
\text { can en la relación con el usuario facilitándose el proceso de evaluación. }\end{array}$ \\
\hline & 04 & Cumplen con eficiencia la recolección de información. \\
\hline & 01 & Destacan en socializar los resultados de la evaluación al resto del equipo. \\
\hline \multirow[b]{2}{*}{ Planificación } & 05 & Al finalizar la práctica logran planificar adecuadamente. \\
\hline & 01 & No hay dificultad en la identificación del problema. \\
\hline \multirow{3}{*}{ Intervención } & 05 & Se destaca la excelente relación terapéutica lograda con cada uno de los usuarios. \\
\hline & 02 & Destaca el compromiso y la responsabilidad profesional. \\
\hline & 01 & Tienen manejo terapéutico y conservan la distancia terapéutica. Gran ética. \\
\hline Solución /Problemas & 01 & Buscan bibliografía adecuada para capacitarse en los aspectos teóricos carentes. \\
\hline \multirow{6}{*}{$\begin{array}{l}\text { Profesionalismo } \\
\text { Administrativo }\end{array}$} & 13 & $\begin{array}{l}\text { Muy buen desempeño administrativo y cumplimiento de las normas con relación a } \\
\qquad \text { la Institución y Profesor Guía. }\end{array}$ \\
\hline & 01 & $\begin{array}{c}\text { Destaca su capacidad empática y su vocación de servicio hacia los usuarios. Man- } \\
\text { tienen unas excelentes relaciones interpersonales con el profesor y el } \\
\text { personal de servicio. }\end{array}$ \\
\hline & 01 & No discriminan a las personas por su situación económica. \\
\hline & 01 & Destacan por sus habilidades relacionales, y su motivación por el trabajo comunitario. \\
\hline & 01 & Acogen las críticas adecuadamente. \\
\hline & 01 & $\begin{array}{c}\text { Siempre están dispuestos a realizar actividades anexas a su práctica, en directo } \\
\text { beneficio con los usuarios. }\end{array}$ \\
\hline
\end{tabular}

En cuanto a la distribución de la frecuencia de mención de las respuestas abiertas, codificadas de acuerdo al análisis del contenido referido, por los Profesores Guía para cada una de las variables de la Competencia profesional, los resultados, en cuanto a las debilidades observadas en el desempeño de los estudiantes, destacan las siguientes: 
Tabla $N^{\circ} 4$

Debilidades de las competencias profesionales

\begin{tabular}{|c|c|c|}
\hline Competencia & Profesores & Respuesta Abierta \\
\hline \multirow{4}{*}{ Evaluación } & 03 & $\begin{array}{l}\text { Deben conocer más pautas o técnicas de evaluación específicas y validadas, rango } \\
\text { articular, pruebas identificados, familia, redes. }\end{array}$ \\
\hline & 02 & Falta conocimientos teóricos para realizar análisis/ interpretación de datos relevantes. \\
\hline & 01 & No hay iniciativa en el desarrollo de informes escritos y no hay claridad en la redacción. \\
\hline & 01 & $\begin{array}{l}\text { Los alumnos contaban con una buena cantidad de baterías de evaluación, pero a ve- } \\
\text { ces las aplicaban todas, aun cuando no era necesario. }\end{array}$ \\
\hline \multirow{7}{*}{ Planificación } & 02 & Tienen dificultad en la elección de los objetivos. \\
\hline & 01 & $\begin{array}{l}\text { Planifican objetivos poco difíciles de alcanzar considerando la realidad de familia. } \\
\text { En relación a los aportes al equipo, hay una tendencia a tratar de abarcar más de lo } \\
\text { que corresponde al Terapeuta Ocupacional. }\end{array}$ \\
\hline & 01 & Falta de síntesis al definir los objetivos a alcanzar en el periodo de estadía de los usuarios. \\
\hline & 01 & A muchos de los alumnos les costaba encontrar un marco teórico de referencia apropiado. \\
\hline & 01 & $\begin{array}{l}\text { Falta incorporar los objetivos de rehabilitación y reinserción laboral. Olvidan el enfo- } \\
\text { que laboral, el análisis ergonómico y biomecánico del puesto de trabajo. }\end{array}$ \\
\hline & 01 & A muchos de los alumnos les costaba encontrar un marco teórico de referencia apropiado. \\
\hline & 01 & $\begin{array}{l}\text { Es necesario profundizar en aspectos técnicos de la redacción y opera lización } \\
\text { de los objetivos. }\end{array}$ \\
\hline \multirow{4}{*}{ Intervención } & 01 & $\begin{array}{l}\text { Poca creatividad por miedo a equivocarse. Falta seguridad en los } \\
\text { conocimientos adquiridos. }\end{array}$ \\
\hline & 01 & $\begin{array}{l}\text { Falta iniciativa para dejar información o instrucciones escritas a las Técnicos en Párvu- } \\
\text { los acerca de la prevención de conductas riesgosas o mantener el plan de tratamiento } \\
\text { cuando los pacientes no van a Terapia Ocupacional. }\end{array}$ \\
\hline & 01 & $\begin{array}{c}\text { Hasta el año } 2004 \text { los alumnos no saben hacer órtesis. No incorporan el } \\
\text { reintegro laboral del paciente. }\end{array}$ \\
\hline & 01 & Falta proyectar la intervención a ambientes normalizadores. \\
\hline \multirow[b]{2}{*}{ Solución /Problemas } & 04 & $\begin{array}{l}\text { El tiempo de dedicación a la práctica, un factor de dificultad para desarrollar a } \\
\text { cabalidad el proceso. }\end{array}$ \\
\hline & 01 & $\begin{array}{l}\text { Los conocimientos teóricos se deben reforzar, lo que lleva al retraso en la adquisición } \\
\text { de habilidades más complejas en el manejo de pacientes. }\end{array}$ \\
\hline $\begin{array}{l}\text { Profesionalismo/ } \\
\text { Administrativo }\end{array}$ & 00 & \\
\hline
\end{tabular}


En este aspecto también se concluye que la dimensión de la variable "Profesionalismo / Administrativo", es la competencia con el nivel mayor de logro obtenido, ya que más del $90 \%$ de los Profesores Guía establecen esta competencia con un total de 4 puntos; lo que significa que los estudiantes en un breve tiempo presentan una respuesta emocional favorable hacia los usuarios, de acuerdo con la percepción y valoración del bienestar de ellos y una gama de sentimientos de simpatía y cariño más que aceptable para este tipo de trabajo.

Cabe destacar que en esta dimensión de la variable "Competencias Profesionales", los Profesores Guía no consignan debilidades de los estudiantes.
Respecto del quinto objetivo específico, cuya meta era verificar y describir cuantitativamente, por área de especialidad en salud de la Terapia Ocupacional, el nivel de aprobación de las competencias profesionales iniciales propias del ejercicio profesional del estudiante que egresa, el año 2004, de la carrera de Terapia Ocupacional de la UPLACED de Valparaíso, la presente investigación, considerando los casos válidos, que se adscriben al criterio aprobado, a partir de la calificación 3 en cada ítem, los Profesores Guía de Práctica Profesional han determinado que estas han sido logradas por los egresados en todas las áreas de especialidad, a excepción de la dimensión "Planificación e Intervención" en el área de la Pediatría, tal como se muestra en la Tabla siguiente.

Tabla $N^{\circ} 5$

Nivel de aprobacion de los profesores guía según área de especialidad en terapia ocupacional

\begin{tabular}{|c|c|c|c|c|c|}
\hline & & $\begin{array}{c}\text { Salud Física } \\
\text { Adulto }\end{array}$ & $\begin{array}{c}\text { Salud Mental } \\
\text { y Psiquiatría }\end{array}$ & $\begin{array}{c}\text { Geriatría y } \\
\text { Gerontología }\end{array}$ & Pediatría \\
\hline \multirow{2}{*}{ Evaluación } & Media & 3.400 & 3.725 & 3.367 & 3.143 \\
& Des Estándar & .4183 & .7741 & 1.0263 & .8304 \\
\hline \multirow{2}{*}{ Planificación } & Media & 3.280 & 3.537 & 3.267 & 2.614 \\
& Des Estándar & .4970 & .9054 & 1.2583 & .8533 \\
\hline \multirow{2}{*}{ Intervención } & Media & 3.500 & 3.625 & 3.200 & 2.986 \\
& Des Estándar & .4000 & 1.1209 & .7211 & .7198 \\
\hline \multirow{2}{*}{ Solución de Problemas } & Media & 3.080 & 3.475 & 2.967 & 3.129 \\
& Des Estándar & .6611 & .9316 & .4041 & .9394 \\
\hline \multirow{2}{*}{ Profesionalismo Administra- } & Media & 4.020 & 4.575 & 3.500 & 3.657 \\
& Des Estándar & .6140 & .5676 & .5000 & .7044 \\
\hline tivo & Media & 3.440 & 3.788 & 3.267 & 3.114 \\
& Des Estándar & .3975 & .7492 & .7638 & .7267 \\
\hline \multirow{2}{*}{ Escala Total } & \multicolumn{2}{|c|}{}
\end{tabular}

Respecto del sexto objetivo específico, cuya meta era verificar y describir cuantitativamente el nivel de aprobación de los Profesores Guía de Práctica Profesional respecto de la formación de competencias iniciales propias del ejercicio profesional del estudiante que egresa el año 2004 de la carrera de Terapia Ocupacional de la UPLACED de Valparaíso, según los años de servicio de este profesional en el desempeño de la profesión, se aplicó el Test de análisis de varianza (ANOVA), cuyos resultados permitieron concluir lo siguiente: Bajo la hipótesis estadística de igualdad de las medias de las escalas entre el grupo que posee entre 5 y 20 años de experiencia profesional como Terapeuta Ocupacional, versus el que posee 21 años y más, no se rechaza la hipótesis de igualdad de medias para todas las escalas y la escala total ANOVA a un nivel de sig- 
nificancia del 5\%. Es decir los años de ejercicio de la profesión, no son un factor determinante en los puntajes medios en cada escala y la escala total.

Respecto del séptimo objetivo específico, cuya meta era verificar y describir cuantitativamente, el nivel de aprobación de los Profesores Guía de Práctica Profesional, respecto de la formación de competencias iniciales propias del ejercicio profesional del estudiante que egresa el año 2004 de la carrera de Terapia Ocupacional de la UPLACED de Valparaíso, según los años de ejercicio como docente de la educación superior, se aplicó el Test de análisis de varianza (ANOVA), cuyos resultados permitieron concluir lo siguiente:

Bajo la hipótesis estadística de igualdad de medias de las escalas entre el grupo que posee entre 0 y 4 años de docencia, versus el que posee 5 años y más, no se rechaza la hipótesis de igualdad de medias para todas las escalas y la escala total ANOVA a un nivel de significancia del 5\%. Es decir los años de docencia no es un factor determinante en los puntajes medios en cada una de las escalas y la escala total.

\section{Discusión de los Resultados}

Como se puede apreciar; la evaluación de competencias es un proceso complejo, que se estructura en torno a conocimientos, habilidades y a las destrezas individuales y sociales de los egresados, debiendo establecer instrumentos de medición que sean válidos y confiables para evidenciar su desempeño en el ámbito laboral. Solo en estas circunstancias se puede aportar al cuerpo de conocimientos basado en el diseño curricular e implementar una cultura de calidad de la formación profesional, que fue el objetivo que orientó este estudio.

En concordancia, también, con De Las Heras C. y Gómez L. S. et al. (2000), "la identificación de los elementos que conforman las competencias que se presentan en una actividad de servicio" como es la Terapia Ocupacional aplicada a los distintos ámbitos de la salud, se debe situar en el territorio de lo observable en términos conductuales, llegando a considerar como unidad de soporte "la comprensión del desempeño ocupacional del alumno (a) en situaciones de bienestar y en caso de las disfunciones ocasionadas por causas psicobiológicas o conductuales". De Las Heras C. G. y Gómez L. S. et al. (2000). Del mismo modo los resultados de esta investigación concuerdan con lo mencionado en el Marco de Trabajo para la Práctica de la Terapia Ocupacional de la Asociación Americana de Terapeutas Ocupacionales (2008), donde se enfatiza que este Marco de Trabajo se construye a partir de dos grandes áreas: la del "dominio" que destaca los alcances de la profesión y el cuerpo de conocimientos de sus profesionales; los que se entrelazan con el nivel de "proceso" que está centrado en el cliente que recibe los servicios del terapeuta ocupacional en una relación transaccional (Gómez L. Silvia y De las Heras D. Carmen, 2009).

El presente estudio se centró en el enfoque de competencias. contribuyendo con la identificación de las competencias profesionales iniciales de los egresados del Programa de Formación Profesional de Terapia Ocupacional de la UPLA el año 2004, al diseñar un instrumento de medición que permite evaluar el desempeño profesional en las distintas áreas de la salud donde el Terapeuta Ocupacional se inserta, desarrollando acciones de prevención, tratamiento y rehabilitación de la salud a través de la participación en ocupaciones, haciendo uso de sus conocimientos para efectuar evaluaciones e intervenciones, reflexionando acerca del usuario/cliente de modo integral, considerando además el medio ambiente que lo rodea.

El instrumento diseñado, logra agrupar las competencias genéricas que constituyen el Perfil de competencias del cargo de Terapeuta Ocupacional, ya que refleja las funciones que le son propias en las diversas áreas de desempeño de este y en su defecto facilitan la gestión de recursos humanos de un modo integral.

Al respecto se coincide con Irigoin, M. y Vargas, F. (2002), en el hecho de que un instrumento de evaluación estratégico es aquel que se concibe en función del "mejoramiento de la calidad de la gestión y más precisamente de la calidad del aprendizaje" (Irigoin, M. y Vargas, F. 2002, pág. 4), cuyos fines, tal como son concebidos en este estudio son acceder a una retroalimentación pertinente y oportuna tanto con relación al logro de los objetivos de la formación profesional, como a la adecuación de estos a los requerimientos de los usuarios y de la sociedad, respecto del perfil epidemiológico diagnosticado por el Estado; el cual está representado en las metas sanitarias gubernamentales.

Por otra parte un instrumento estandarizado y validado como ocurre con el que ha sido diseñado para este estudio, logra establecer que los atributos propios del Profesor(a) Guía (años de experiencia profesional y de docencia en Instituciones de Educación Superior), no constituyen un cambio significativo a la hora de evaluar los desempeños de los estudiantes. 
Establecido lo anterior, queda de manifiesto que dicho instrumento de evaluación de las Prácticas Profesionales de la carrera de Terapia Ocupacional de la UPLA, facilita el seguimiento de los estudiantes derivados a través de convenios Interinstitucionales a lo largo del país, ya que permite prescindir de la presencia del supervisor de práctica, figura común de distintas casas de estudios superiores, que encarece la formación profesional, y dificulta la salida al trabajo de campo en la etapa final del Programa de Formación.

En cuanto a sus resultados, el Programa de Formación Profesional de Terapeutas Ocupacionales de la UPLA, se puede concluir que responde en forma adecuada a las necesidades del mercado laboral en la mayoría de las áreas donde se desempeñan sus egresados; dado que tal como Le Bortef (2013) declara, los egresados de la Carrera de Terapia Ocupacional de la UPLA, al término de su Práctica profesional saben actuar pertinentemente y con competencia en las distintas situaciones de trabajo donde les corresponde abordar problemas de salud.

No obstante lo anterior, dados los resultados, queda como tarea pendiente la revisión y ajuste de la línea de desarrollo profesional de la Terapia Ocupacional en Infancia Temprana y la Pediatría acorde a las necesidades que los usuarios de los servicios de salud requieren hoy.

Por otra parte se hace necesario declarar las limitaciones propias de un estudio mixto con seres humanos, y es la necesidad de replicarlo varias veces para poder controlar variables intervinientes que permitan generalizar los resultados y aportar sistemáticamente a la cultura de la calidad.

Otro elemento necesario de mencionar para el avance en la construcción de una línea de educación de calidad es el necesario trabajo en conjunto con el medio donde se insertan los futuros profesionales Terapeutas Ocupacionales, ya que permite una retroalimentación permanente del cumplimiento del Perfil profesional de los egresados. Si bien el trabajo en equipo con los Profesores Guía de Práctica Profesional fue una política desarrollada a los inicios de la Carrera de la UPLA, estas reuniones fueron descontinuadas, quedando de manifiesto hoy la necesidad de retomarlas a la luz de los resultados obtenidos en la formación de los estudiantes en el área del desarrollo en la infancia como también en la Pediatría.

En síntesis, los aportes de la investigación se centran en los siguientes aspectos: la evidencia de la necesidad de construir nuevos mecanismos para ampliar y fortalecer el vínculo entre la formación académica y la formación profesional, la necesidad de modificar los contenidos del Programa de Formación Profesional en el nuevo diseño curricular basado en competencias, la importancia de la evaluación pedagógica en el contexto de la evaluación permanente, la evidencia del logro de los objetivos del Programa de Formación Profesional de los estudiantes del año 2004 en un 80,56\% y por último el haber diseñado el primer Instrumento de Evaluación de las Competencias Profesionales iniciales de los Terapeutas Ocupacionales, validado en el país.

\section{ReferenCiAs BibliográficAs}

Cejas, E., Pérez, J. (2013) Mag.(c). (ciudad de la habana. ISPETD. Disponible en http/:monografias.com). Un concepto muy controvertido: Competencias Laborales.

De Las Heras C. G. y Gómez L. S. et al. (2000). Posición Sobre la Formación Profesional. Santiago: Colegio de Terapeutas Ocupacionales de Chile (inédito).

Gómez, S. \& De Las Heras C. Práctica de Terapia Ocupacional: Marco de Trabajo: dominio y proceso. $2^{\text {a }}$ edición, 2009. Recuperado el 30 de octubre de 2012 de www.es.scrib.com/ doc/70966853/Marco-de-Trabajo2-Revisado-2010

Guy, Le Bortef (23 de enero de 2013). Tener Competencias no significa ser competente. Universidad de Colima, México. Recuperado el 18 de junio de 2013. de http://www.ucol.mx/noticias_boletin.php?id=12805 .

Hernández S.R. et al. (2003). Metodología de la Investigación. Análisis de los datos. (p. 595). México: Mc Graw Hill (3a ed.).

Irigoin, M. y Vargas, F. (2002). Competencia laboral: Manual de conceptos, métodos y aplicaciones en el Sector Salud. (Programa de desarrollo de recursos humanos. División de Sistemas y Servicios de Salud de la Organización Panamericana de la Salud -OPS / OMS). Prólogo. Montevideo: Centro Interamericano de Investigación y Documentación sobre Formación Profesional de la Oficina Internacional del Trabajo (CINTERFOR / OIT, Primera Edición).

Latrach A. Cecilia, Soto F. Paz, González V. Irene y otros. Aseguramiento de la calidad en la formación de las enfermeras desde la perspectiva de los procesos de acreditación nacional. Ciencia y Enfermería, vol. 15 (n.2), pp. 79-94. 2009. Recuperado el 27 de abril de 2012 de http://www.scielo.cl/pdf/cienf/ v15n2/art09.pdf

Smith H. Evaluación total y evaluación específica en Terapia Ocupacional: consideraciones generales. Unidad II. Capítulo 7. Willard y Spackman: "Terapia Ocupacional" 08 a edición.

Editorial Médica Panamericana. 2001. Traducción: Koury, S. Padilla, S. Reinoso, G. y Kljan, D. 